\title{
Tazarotene-Induced Gene 1 Enhanced Cervical Cell Autophagy through Transmembrane Protein 192
}

\author{
Rong-Yaun Shyu ${ }^{1}$, Chun-Hua Wang ${ }^{2}$, Chang-Chieh $\mathrm{Wu}^{3}$, Mao-Liang Chen ${ }^{4}$, Ming-Cheng Lee ${ }^{4}$, Lu-Kai \\ Wang $^{5}$, Shun-Yuan Jiang ${ }^{4, *}$, and Fu-Ming Tsai ${ }^{4, *}$
}

Tazarotene-induced gene 1 (TIG1) is a retinoic acidinducible protein that is considered a putative tumor suppressor. The expression of TIG1 is decreased in malignant prostate carcinoma or poorly differentiated colorectal adenocarcinoma, but TIG1 is present in benign or welldifferentiated tumors. Ectopic TIG1 expression led to suppression of growth in cancer cells. However, the function of TIG1 in cell differentiation is still unknown. Using a yeast two-hybrid system, we found that transmembrane protein 192 (TMEM192) interacted with TIG1. We also found that both TIG1A and TIG1B isoforms interacted and co-localized with TMEM192 in HtTA cervical cancer cells. The expression of TIG1 induced the expression of autophagy-related proteins, including Beclin-1 and LC-3B. The silencing of TMEM192 reduced the TIG1-mediated upregulation of autophagic activity. Furthermore, silencing of either TIG1 or TMEM192 led to alleviation of the upregulation of autophagy induced by all-trans retinoic acid. Our results demonstrate that the expression of TIG1 leads to cell autophagy through TMEM192. Our study also suggests that TIG1 and TMEM192 play an important role in the all-trans retinoic acid-mediated upregulation of autophagic activity.

\section{INTRODUCTION}

Tazarotene-induced gene 1 (TIG1), also known as retinoic acid receptor responder 1 (RARRES1), was first identified in skin raft cultures treated with the RAR $\beta / Y$-selective retinoid $A G N$

\footnotetext{
${ }^{1}$ Department of Internal Medicine, ${ }^{2}$ Department of Dermatology, Taipei Tzuchi Hospital,The Buddhist Tzuchi Medical Foundation, New Taipei City 231, Taiwan, ${ }^{3}$ Department of Surgery, Tri-Service General Hospital, National Defense Medical Center, Taipei 114, Taiwan, ${ }^{4}$ Department of Research, Taipei Tzuchi Hospital,The Buddhist Tzuchi Medical Foundation, New Taipei City 231, Taiwan, ${ }^{5}$ Radiation Biology Core Laboratory, Institute for Radiological Research, Chang Gung University/Chang Gung Memorial Hospital, Linkou, Taoyuan 333, Taiwan

*Correspondence: jiang.shunyuan@gmail.com (SYJ); afu2215@gmail.com (FMT)
}

Received 28 June, 2016; revised 15 November, 2016; accepted 18 November, 2016; published online 13 December, 2016

Keywords: all-trans retinoic acid, autophagy, Beclin-1, LC3B, tazaroteneinduced gene 1, transmembrane protein 192
190168, which is also called tazarotene (Nagpal et al., 1996). The TIG1 gene, which is located on chromosome 3q25.32, encodes two isoforms: TIG1A and TIG1B. These two isoforms are encoded by a 1.55-kb mRNA [GenBank: NM_206963] and an 883-bp mRNA [GenBank: NM_002888], which can be translated into proteins consisting of 294 amino acids with a molecular weight of $33.3 \mathrm{kDa}$ or 228 amino acids with a molecular weight of $25.8 \mathrm{kDa}$, respectively.

TIG1 is structurally similar to the protein latexin with an Nterminal transmembrane domain ranging from 21 to 42 amino acids that targets the protein to endomembranes (Liang et al., 2007). Although latexin is considered a carboxypeptidase inhibitor, TIG1's proteolytic activity remains unknown. TIG1 suppressed the growth and invasion of several types of cancer cells, and CpG hypermethylation of the TIG1 promoter led to downregulation of TIG1 expression in various carcinomas (Chen et al., 2014; Jing et al., 2002; Kwok et al., 2009; Kwong et al., 2005; Mizuiri et al., 2005; Peng et al., 2012; Shutoh et al., 2005; Wu et al., 2006; Yanatatsaneejit et al., 2008; Zhang et al., 2004). Expression of TIG1 is decreased in malignant prostate carcinoma cell lines and in poorly differentiated colorectal adenocarcinoma, but TIG1 is present in benign or welldifferentiated tumor lines (Jing et al., 2002; Wu et al., 2006), and ectopic TIG1 expression led to suppression of growth in cancer cells (Jing et al., 2002; Tsai et al., 2011; Wu et al., 2011). Therefore, TIG1 might play an important role in retinoic acidmediated cellular differentiation and tumor growth suppression.

The transmembrane protein 192 (TMEM192) gene is located on chromosome 4q32.3 and encodes a protein with 271 amino acids and a molecular weight of $30.9 \mathrm{kDa}$. TMEM192 is a lysosomal membrane-bound protein that is widely expressed in human kidney, liver, lung, and pancreas tissue (Schroder et al., 2010). Deficiency of TMEM192 in hepatoma HepG2 cells results in growth inhibition and increased apoptosis. Increased apoptosis in TMEM192-deficient cells was inhibited by blocking the autophagy gene Atg7, suggesting that TMEM192 plays an important role in autophagy and may contribute to the regulation of cell apoptosis (Liu et al., 2012).

Autophagy is a conserved lysosomal degradation pathway that controls the quality of the cytoplasm by destroying disassembled proteins and unnecessary or dysfunctional cellular components (Kobayashi, 2015; Lee et al., 2012; Mizushima and Komatsu, 2011). Autophagy is regulated by autophagyrelated (Atg) genes that control the formation and maturation of double-membrane vesicles called autophagosomes, which engulf and transport cellular proteins and organelles. Autopha- 
gosomes subsequently fuse with lysosomes to form autolysosomes and then digest the contents with acid hydrolases provided by the lysosome (Lamb et al., 2013; Mizushima et al., 2011). Many Atg genes are involved in autophagosome formation, including the well-known Atg12-Atg5 and Atg8 (microtubule-associated protein 1 light chain 3, LC3)-phosphatidylethanolamine systems (Dooley et al., 2014; Hanada et al., 2007). ULK (the mammalian homolog of Atg1) and Beclin-1 (mammalian homolog of Atg6) have been shown to contribute to the activation of downstream autophagy components (Hara et al., 2008; Kang et al., 2011; Russell et al., 2013).

To ascertain the biological functions of TIG1, we screened novel interacting proteins of TIG1B using a yeast two-hybrid system. Our results showed that TIG1B directly binds to TMEM192. TIG1A shares the N-terminal 224 amino acids with TIG1B, suggesting that TIG1A may also interact with TMEM192. TMEM192 is considered a lysosomal protein that is involved in the autophagic process. In this study, we demonstrated a role for TIG1 in the induction of autophagy activity in cervical cancer cells via the TMEM192 protein.

\section{MATERIALS AND METHODS}

\section{Yeast two-hybrid screening}

Plasmids pAS2-1 and pACT2 and yeast strains Y187 and CG1945 were purchased from Clontech Laboratories (USA). To generate an expression vector for the DNA-binding domain of the transcriptional activator GAL4 (GAL4 BD)-tagged cytoplasmic region of TIG1B, truncated TIG1B cDNA fragments were amplified from pTIG1B-myc using 5' (5'- CCGAATTCCCCGAC GACCCTGGGCAGCC -3') and 3' (5'- ACGGATCCTCAGGTT TTTCTTACCCACTGCCTCACAC $-3^{\prime}$ ) primers and then subcloned in-frame into the EcoRl-BamHI sites of pAS2-1 to generate pGAL4 BD-TIG1B ${ }_{43-228}$. The plasmid pAS2-1/TIG1B ${ }_{43-228}$ was used as bait for screening a HeLa cell cDNA library that was fused to the GAL4 activation domain (AD) vector PACT2 in yeast strain CG-1945 (Trp/Leu'). Histidine-positive colonies were then assayed for $\beta$-galactosidase activity. Plasmids from $\mathrm{His}^{+} / \mathrm{LacZ}^{+}$clones were isolated from the yeast and then transformed into E. coli strain HB101 for amplification. These cDNAcontaining Y187 yeast were then mated with CG-1945, which

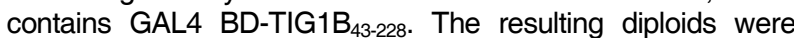
plated on medium without tryptophan, leucine, or histidine, and $\beta$-galactosidase activity was determined. Clones containing CDNA for possible TIG1-binding proteins were screened by PCR. The amplified products were analyzed on $1 \%$ agarose gels to estimate the size of the products.

\section{Expression vectors}

The vector pEGFP-LC3 (human) was a gift from Toren Finkel (Addgene plasmid \# 24920) (Lee et al., 2008). The vectors pTIG1A-myc and pTIG1B-myc have been described previously (Wu et al., 2011). To generate pTMEM192-Flag, the TMEM192 cDNA fragment was amplified from pTMEM192/PACT2 using 5' (5'-TGGCTAGCATGGCGGCGGGGGGCAGGATG-3') and 3' (5'-CGGAATTCGCGTTCTACTTGGCTGACAGCCC-3') primers and then subcloned in-frame into the Nhel-EcoRl sites of PCR3.1-Flag (Dr. Yi-Ling Lin, Institute of Biomedical Science, Academia Sinica, Taipei, Taiwan). To generate pEGFP-TMEM192, the TMEM192 cDNA fragment was amplified from pTMEM192Flag using 5' (5'-GACTCGAGCTATGGCGGCGGGGGGCAG GATG-3') and $3^{\prime}$ (5'-GTGGATCCCGTTCTACTTGGCTG ACAGCCCAGG-3') primers and then subcloned in-frame into the Xhol-BamHI sites of the pEGFP-C1 vector (Clontech).
Cell culture and transfection

HeLa Tet-off (HtTA) cervical cancer cells were maintained in RPMI-1640 medium supplemented with 25 mM HEPES, 26 $\mathrm{mM} \mathrm{NaHCO}$, $2 \mathrm{mM}$ L-glutamine, penicillin (100 units $/ \mathrm{mL}$ ), streptomycin $(100 \mu \mathrm{g} / \mathrm{ml})$, and $10 \%$ fetal bovine serum (FBS) at $37^{\circ} \mathrm{C}$ in $5 \% \mathrm{CO}_{2}$. The hepatoma-derived cell line Huh7 was a generous gift from Dr. C.-S. Hsu (Department of Internal Medicine, Taipei Tzuchi Hospital, The Buddhist Tzuchi Medical Foundation, Taiwan). Cells were grown in Dulbecco's modified Eagle's medium supplemented with 10\% FBS, $2 \mathrm{mM}$ Lglutamine, 100 units $/ \mathrm{ml}$ penicillin, and $10 \mu \mathrm{g} / \mathrm{ml}$ streptomycin at $37^{\circ} \mathrm{C}$ under $5 \% \mathrm{CO}_{2}$ in a $95 \%$ humidified atmosphere. Cells plated in culture dishes were transfected with the expression vectors using liposome-mediated transfection. Briefly, plasmids and Lipofectamine 2000 (Gibco BRL, USA) were diluted in Opti-MEM medium and then mixed with plasmids at room temperature for $15 \mathrm{~min}$. The DNA-Lipofectamine complexes were then added to cells for $5 \mathrm{~h}$ at $37^{\circ} \mathrm{C}$. Cells were refreshed with complete medium for $24-48 \mathrm{~h}$ at $37^{\circ} \mathrm{C}$ for further analysis.

\section{Immunoprecipitation and Western blotting}

Cells were lysed in modified RIPA buffer (20 mM Tris [pH 7.5], $100 \mathrm{mM} \mathrm{NaCl}, 1 \% \mathrm{NP}-40,30 \mathrm{mM}$ sodium pyrophosphate) containing a protease inhibitor cocktail (Roche Diagnostics, Germany) and phosphatase inhibitors. Cell lysates containing $500 \mu \mathrm{g}$ of protein were first incubated for $2 \mathrm{~h}$ at $4^{\circ} \mathrm{C}$ with $3.2 \mu \mathrm{g}$ of anti-MYC (Invitrogen, USA) or anti-FLAG (Sigma, USA) monoclonal antibody and then incubated for $2 \mathrm{~h}$ at $4^{\circ} \mathrm{C}$ with 20 $\mu \mathrm{l}$ of protein G plus/protein A-agarose (Calbiochem, USA). Immunoprecipitated complexes were analyzed by Western blotting using an anti-FLAG or anti-MYC antibody after the complexes were washed three times with PBS. Alternatively, cell lysates containing $5 \mathrm{mg}$ of protein were incubated first with $8 \mu \mathrm{g}$ of normal mouse IgG (Santa Cruz Biotechnology, USA) or $8 \mu \mathrm{g}$ of anti-TIG1 (Santa Cruz Biotechnology) antibody for $12 \mathrm{~h}$ at $4^{\circ} \mathrm{C}$ and then incubated with $20 \mu$ of protein G plus/protein $A$ agarose at $4^{\circ} \mathrm{C}$ for $2 \mathrm{~h}$. Immunoprecipitated complexes were washed three times with modified RIPA buffer and then analyzed by Western blotting using anti-TMEM192 (Santa Cruz Biotechnology) antibody. For Western blotting, 20-60 $\mu \mathrm{g}$ of proteins was separated on $15 \%$ polyacrylamide gels and transferred to polyvinylidene fluoride membranes. After the membranes were blocked, they were incubated for $12 \mathrm{~h}$ at $4^{\circ} \mathrm{C}$ with anti-MYC, anti-FLAG, anti-TIG1 (Santa Cruz Biotechnology), anti-TMEM192 (Santa Cruz Biotechnology), anti-LC3B (Cell Signaling Technology, USA), anti-SQSTM1/p62 (Cell Signaling Technology), anti-Beclin-1 (Cell Signaling Technology), or antiactin (Sigma) antibody and then incubated with horseradish peroxidase-conjugated goat anti-mouse antibody at room temperature for $1 \mathrm{~h}$. An ECL kit (Amersham, UK) was used to detect the substrate reaction.

\section{Confocal and immunofluorescence analysis} HtTA and Huh7 cells $\left(1 \times 10^{5}\right)$ were plated on poly-L-lysinecoated coverslips in 35-mm dishes in growth medium. Cells were then transfected with $500 \mathrm{ng}$ of TIG1A-myc or TIG1B-myc expression vector along with $500 \mathrm{ng}$ pEGFP-TMEM192 expression vector for $18 \mathrm{~h}$. The cells were washed, fixed with $4 \%$ paraformaldehyde and then incubated with anti-MYC antibody and anti-LAMP1 (lysosome-associated membrane protein 1) antibody (Cell Signaling Technology) followed by Alexa Fluor 633 anti-mouse IgG and Alexa Fluor 405 anti-rabbit IgG antibodies (Invitrogen). The cells were then analyzed for TIG1A or TIG1B and EGFP-TMEM192 expression with a Leica TCS SP5 
scanner (Leica, Germany). Alternatively, cells were plated in triplicate in 24-well plates at a density of $2 \times 10^{4}$ cells per well in RPMI-1640 medium containing 10\% FBS and incubated overnight. The cells were transfected with $75 \mathrm{ng}$ of TIG1A-myc or TIG1B-myc expression vector or with $75 \mathrm{ng}$ of TMEM192-Flag expression vector along with $150 \mathrm{ng}$ of pGFP-LC3 expression vector and then refreshed with complete medium immediately and $24 \mathrm{~h}$ after transfection. Cells were refreshed in medium without serum and treated with $400 \mathrm{nM}$ rapamycin for $6 \mathrm{~h}$. The cells were washed, fixed with $4 \%$ paraformaldehyde and then analyzed with an Olympus IX-70 immunofluorescence microscope (Olympus, Denmark). The percentage of cells with GFPLC3 puncta formation was calculated by counting the number of cells with punctate GFP-LC3 fluorescence of all GFP-positive cells. A minimum of 100 cells from 3 randomly selected fields were scored.

\section{RNA isolation and quantitative real-time reverse}

transcription polymerase chain reaction (RT-PCR)

Total RNA was extracted using TRIZOL reagent (Invitrogen) according to the manufacturer's instructions. The total RNA was then reverse transcribed to cDNA by incubating at $37^{\circ} \mathrm{C}$ for $1 \mathrm{~h}$ in $20 \mu \mathrm{l}$ of a mixture containing $3 \mu \mathrm{g}$ total RNA, $1 \mathrm{U}$ Moloney murine leukemia virus (MuLV) reverse transcriptase (Invitrogen), $0.5 \mu$ g oligo-d $\mathrm{T}_{12-18}, 4 \mu \mathrm{l} 5 \mathrm{X}$ RT buffer, $0.5 \mathrm{mM} \mathrm{dNTP}$, and $1 \mathrm{U}$ RNaseout recombinant RNase inhibitor. Quantitative realtime PCR (Q-PCR) was performed in triplicate using $20 \mu \mathrm{l}$ of the reaction mixture containing $10 \mu$ Fast SYBR Green Master Mix (Applied Biosystems, USA), $50 \mathrm{ng}$ cDNA, and genespecific forward and reverse primers at $1 \mu \mathrm{M}$ final concentration in a thermal cycler $(7900 \mathrm{HT}$ Fast Real-Time PCR System, ABI). The PCR cycling had an initial incubation at $95^{\circ} \mathrm{C}$ temperature for $3 \mathrm{~min}, 40$ cycles of denaturation at $95^{\circ} \mathrm{C}$ for $15 \mathrm{~s}$ followed by annealing and extension at $60^{\circ} \mathrm{C}$ for $1 \mathrm{~min}$. The PCR primers used for amplification included the following: GAPDH (sense, 5'-GAGTCAACGGATTTGGTCGT-3' and antisense, 5'-TTGAT TTTGGAGGGATCTCG-3'), TIG1 (sense, 5'-CGTGGTCTTCA GCACAGAGCG-3' and antisense, 5'-CCCAAACGTCCCTCAC CTTCC-3'), and TMEM192 (sense, 5'-CAGTCCTCTGGCAAC ACAGT-3' and antisense, 5'-CTGAATCCAGTCTCCGAGGT$\left.3^{\prime}\right)$. The relative expression levels of the TIG1 and TMEM192 cDNA were quantified after normalizing for GAPDH.

\section{RNA interference}

Two TIG1 siRNAs, which are targeted to nucleotides 488-508 and 540-560 of both TIG1A and TIG1B, have been described previously (Wu et al., 2011). TMEM192 siRNA oligonucleotides were synthesized by Sigma. Two TMEM192 siRNAs targeted to nucleotides 441-459 (5'-CCAGUAUCACCACAGCAAAtt-3') and 779-797 (5'-CUAUUUCAAGCCUAGAAGAtt-3') were synthesized based on GenBank accession number NM_001100389.1. The universal control (NC) siRNA (Sigma) was used as a negative control.

\section{Measurement of Beclin-1}

HtTA and Huh7 cells were seeded at $1 \times 10^{5}$ cells per well in 6well plates overnight and then transfected with pTIG1A-myc, pTIG1B-myc expression vector, or control vector for $5 \mathrm{~h}$. Cells were refreshed with complete medium for $24 \mathrm{~h}$ and then incubated in medium without serum for $6 \mathrm{~h}$. Alternatively, cells were transfected with TIG1 siRNA, TMEM192 siRNA, or NC siRNA for $5 \mathrm{~h}$ and then incubated in medium without serum for $6 \mathrm{~h}$ after which they were incubated in growth medium containing all-trans retinoic acid (ATRA) for $48 \mathrm{~h}$. After the cells were washed twice with PBS, they were lysed with modified RIPA buffer, scraped, and collected by centrifugation. The levels of Beclin-1 in the supernatants were determined using a human Beclin-1 ELISA kit according to the manufacturer's instructions (LSBio, Lifespan Biosciences Inc, USA).

\section{Statistical analysis}

Data are represented as the mean \pm SD of at least duplicate studies. Statistical analyses were performed using one-way ANOVA with Dunnett's post hoc test for comparisons of more than two groups. Student's $t$ test was used for comparisons between two groups. A $p$ value $<0.05$ was considered statistically significant.

\section{RESULTS}

TIG1 interacts and co-localizes with the TMEM192 protein We performed yeast two-hybrid screening using the cytoplasmic region of TIG1B as bait and found that TIG1B can interact with TMEM192. To confirm the interaction between TIG1 and TMEM192, we first constructed expression vectors that synthesized recombinant proteins containing Flag-tagged TMEM192. Ectopically expressed recombinant TMEM192-FLAG fusion protein with the expected molecular weight of $35 \mathrm{kDa}$ was observed in HtTA cells after transient transfection for $24 \mathrm{~h}$ (Figs. $1 \mathrm{~A}$ and $1 \mathrm{~B})$. We then performed co-immunoprecipitation using anti-MYC antibody against the MYC epitope of the TIG1A or TIG1B fusion proteins in lysates of TMEM192-Flag-transfected HITA cells. The TIG1A and TIG1B proteins were detected on immunoblots from TMEM192 co-transfected immunoprecipitates, indicating the presence of TIG1A and TIG1B in the TMEM192 pull-down protein complexes prepared in vitro (Fig. 1A). Similarly, TMEM192 was present in the TIG1A or TIG1B immunoprecipitates (Fig. 1B). To validate the endogenous interaction of TIG1 and TMEM192, we performed co-immunoprecipitation, using anti-TIG1 antibody in HtTA cell lysates. Our result revealed that endogenous TIG1 can interact with TMEM192 (Supplementary Fig. 1).

To further verify the co-localization of TIG1 and TMEM192 in situ, we co-transfected HtTA cells with pEGFP-TMEM192 along with pTIG1A-myc or pTIG1B-myc expression vectors for $18 \mathrm{~h}$ and then stained with a lysosomal marker. Both TIG1A and TIG1B were localized with lysosome-specific LAMP1 protein where they colocalized with EGFP-TMEM192 (Fig. 1C). Similar results were observed in Huh7 hepatoma cells, for which TIG1A and TIG1B were primarily distributed at lysosomes, and most of the TIG1A or TIG1B and EGFP-TMEM192 proteins were co-localized in co-transfected Huh7 cells (Supplementary Fig. 2).

\section{TIG1 did not affect TMEM192 protein expression}

Because TIG1 is considered a carboxypeptidase inhibitor (Aagaard et al., 2005), we then examined the effect of TIG1 on TMEM192 mRNA and protein expressions. Upon transfection of TIG1A-myc expression vector for $24 \mathrm{~h}$, TIG1 mRNA level was increased by 4314-fold. Induced expression of TIG1A didn't significantly increased TMEM192 mRNA levels (Fig. 2A). Similar result was observed in cells expressing TIG1B (data not shown). In addition, TMEM192 mRNA level was increased by 3220-fold in cells transfected with TMEM192-flag expression vector. The expression of TIG1mRNA did not differ between control and TMEM192-transfected cells (Fig. 2B). Transfection with 0.5-1.5 $\mu \mathrm{g}$ of TIG1A-myc (Fig. 2C) or TIG1B-myc (Fig. 2D) expression vectors did not considerably affect the expression of 

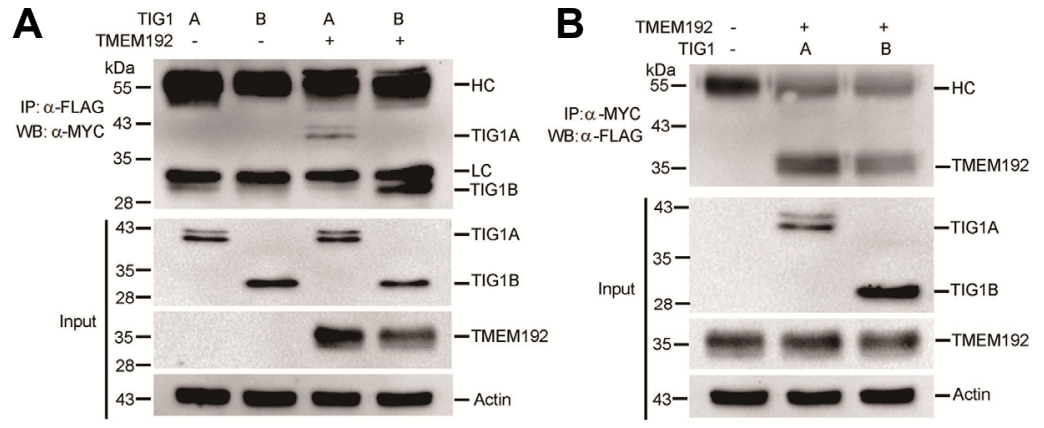

C
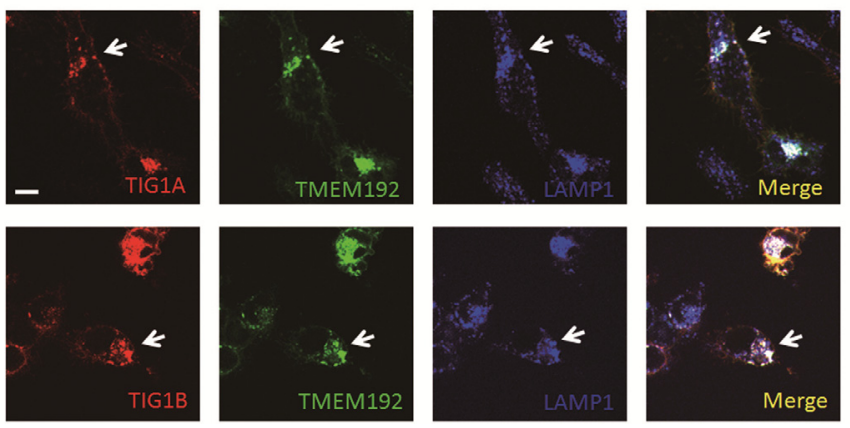

A

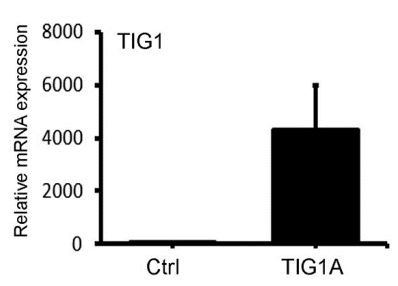

B
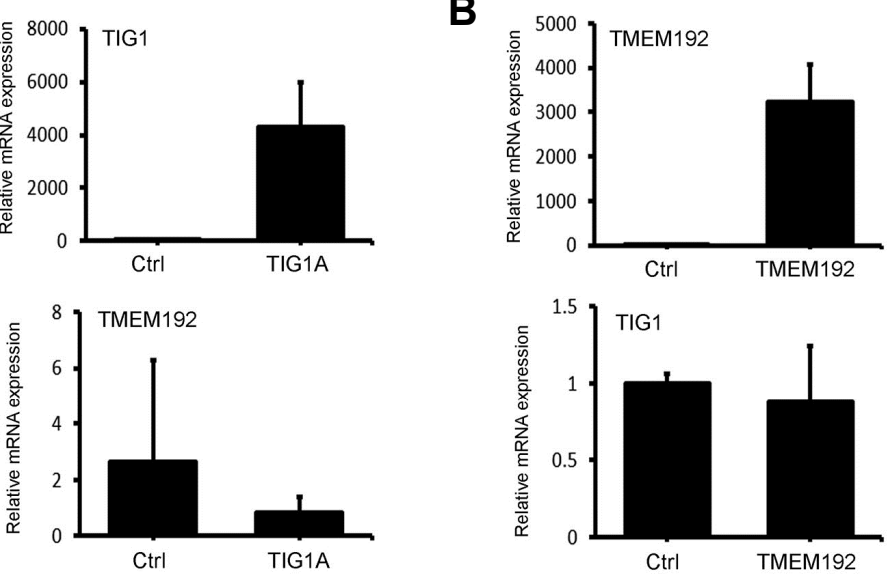

C

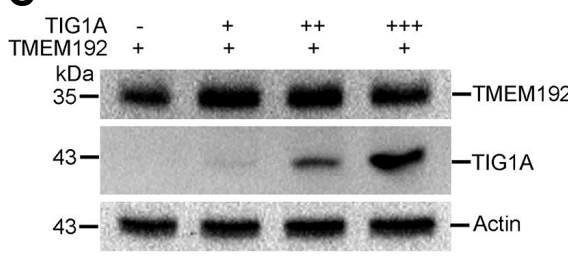

E

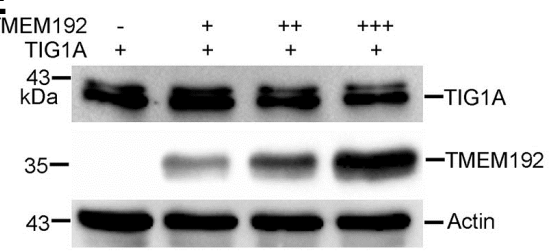

D

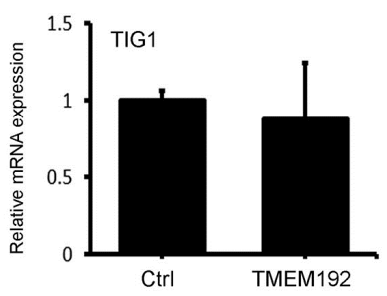

$\mathbf{F}$
Fig. 1. TIG1 interacts and co-localizes with TMEM192. HtTA cells plated in a 10-cm dish were transfected with $3 \mu \mathrm{g}$ of TMEM192-Flag expression vector along with the TIG1A-myc or TIG1B-myc expression vector for $24 \mathrm{~h}$. Cell lysates were prepared, and the interaction between TIG1 and TMEM192 was analyzed by immunoprecipitation followed by Western blot analysis. Immunoprecipitates were resolved by SDS-PAGE and immunoblotted using the anti-MYC antibody (A) or antiFLAG antibody (B). Total cellular extracts from $\mathrm{HtTA}$ cells were subjected to Western blot analysis for TIG1, TMEM192, and actin. HtTA cells were cotransfected with EGFP-TMEM192 along with the TIG1A-myc or TIG1B-myc expression vectors for $18 \mathrm{~h}$. The cells were fixed and then incubated with anti-MYC and anti-LAMP1 (lysosomal marker) antibodies followed by Alexa Fluor 633 goat antimouse IgG and Alexa Fluor 405 goat anti-rabbit IgG antibodies. The cells were then analyzed with a laser scanning confocal microscope. Scale bar: $10 \mu \mathrm{m}$. The localization of TIG1 (red), TMEM192 (green), and lysosomes (blue) was analyzed using a laser scanning confocal microscope. Arrows indicate co-localization of TIG1 and TMEM192 (C). Scale bar, $10 \mu \mathrm{m}$.

Fig. 2. Effects of TIG1 on expression of TMEM192 in HtTA cells. HtTA cells plated in 6well dishes were transfected with $0.5 \mu \mathrm{g}$ of TIG1Amyc (A) or TMEM192-flag (B) expression vector for $24 \mathrm{~h}$. Total RNA was extracted and relative levels of the indicated mRNAs were measured by real-time RT-PCR. HtTA cells plated in a $6-\mathrm{cm}$ dish were transfected with $0.5 \mu \mathrm{g}$ of TMEM192Flag expression vector along with $0.5-1.5 \mu \mathrm{g}$ of TIG1A-myc (C) or TIG1B-myc (D) expression vector for $12 \mathrm{~h}$. Alternatively, cells were transfected with 0.5-1.5 $\mu \mathrm{g}$ of TMEM192-Flag expression vector along with $0.5 \mu \mathrm{g}$ of TIG1A-myc (E) or TIG1B-myc (F) expression vector for $12 \mathrm{~h}$. Cell lysates were prepared, and the levels of TIG1A, TIG1B, and actin were determined by immunoblotting.
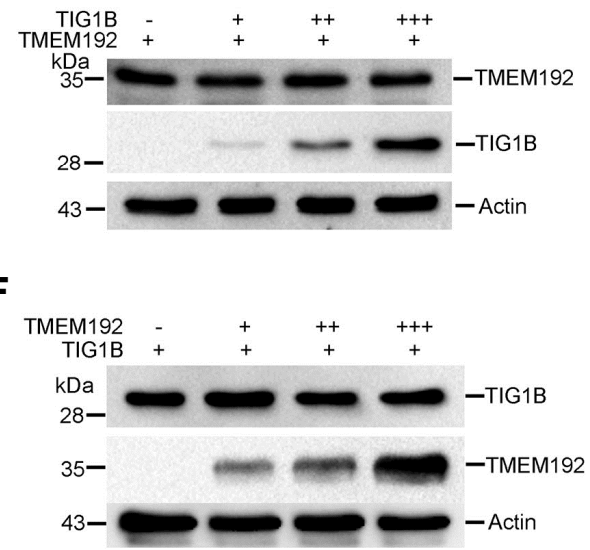
A

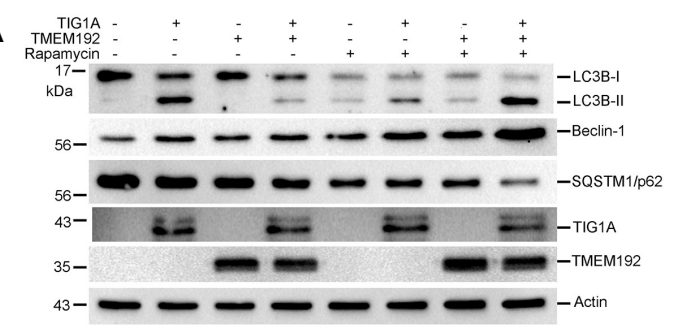

C

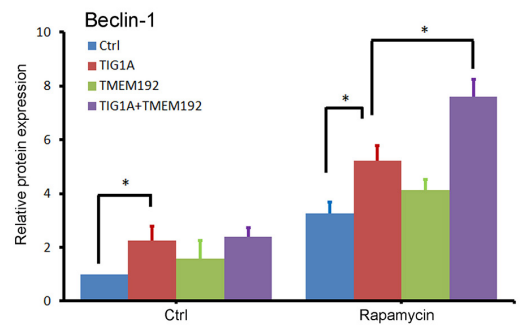

$\mathbf{E}$

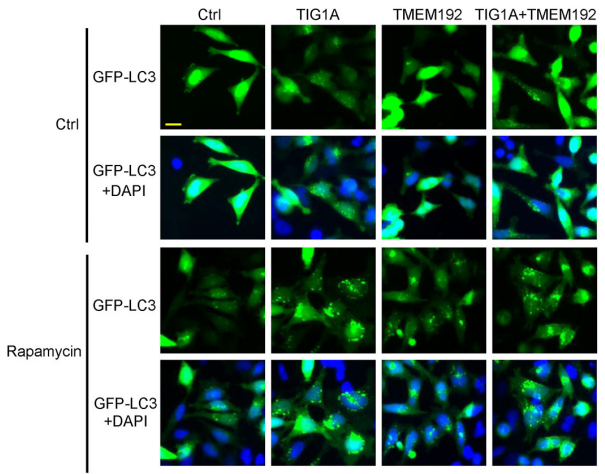

B

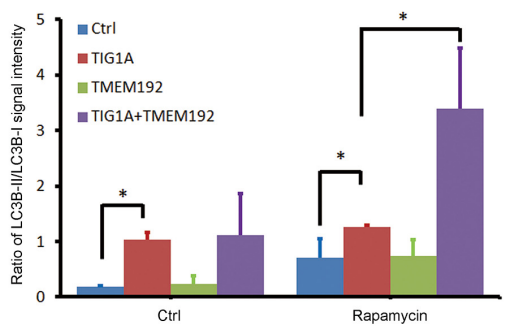

$\mathbf{D}$

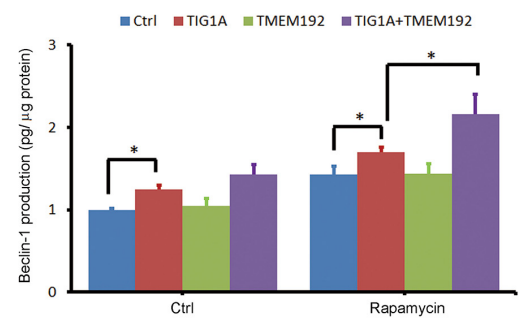

$\mathbf{F}$

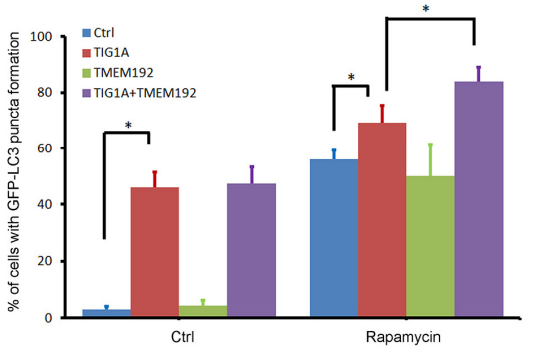

Fig. 3. TIG1A induced the expression of autophagy-related proteins. HtTA cells plated in a 6 -cm dish were transfected with $1.5 \mu \mathrm{g}$ of TMEM192-Flag expression vector along with $1.5 \mu \mathrm{g}$ of TIG1A-myc expression vector for $24 \mathrm{~h}$. Cells were refreshed in medium without serum and treated with $200 \mathrm{nM}$ rapamycin for $6 \mathrm{~h}$. Cell lysates were prepared, and the levels of LC-3B, Beclin-1, SQSTM1/p62, TIG1A, and actin were determined by immunoblotting (A). Experimental results are summarized as the mean percentage $( \pm S D)$ of the ratio of LC3B-II to LC3B$I$ and the level of Beclin-1 with each sample normalized to the level of actin protein in two independent experiments (B, C). HtTA cells plated in 6-well dishes were transfected with $0.5 \mu \mathrm{g}$ of the indicated vectors or the control vector for $24 \mathrm{~h}$ and were then cultured in serum-free medium with $200 \mathrm{nM}$ rapamycin for $6 \mathrm{~h}$. Cell lysates were prepared, and the level of Beclin-1 was detected using an enzyme immunoassay. Representative results of three independent experiments are shown (D). HtTA cells plated in triplicate in 24-well plates were transfected with $75 \mathrm{ng}$ of TIG1A-myc expression vector, $75 \mathrm{ng}$ of TMEM192-Flag expression vector and $150 \mathrm{ng}$ of pGFP-LC3 expression vector for $24 \mathrm{~h}$. Cells were refreshed in medium without serum and treated with $400 \mathrm{nM}$ rapamycin for $6 \mathrm{~h}$. Representative images with GFP-LC3 puncta formation (E). Bar chart indicating the percentage of cells with GFP-LC3 puncta formation from three independent experiments (F). Scale bar, $10 \mu \mathrm{m}$. *Indicates $p$ value $<0.05$

TMEM192 protein, suggesting that TIG1 did not affect the stability of the TMEM192 protein. Similarly, no significant alteration of TIG1A-MYC (Fig. 2E) or TIG1B-MYC (Fig. 2F) protein levels was observed in cells transfected with $0.5-1.5 \mu \mathrm{g}$ of TMEM192Flag expression vectors.

TIG1 induced expression of autophagy-related proteins Since TMEM192 deficiency can induce autophagy (Liu et al., 2012), we next examined whether TIG1 plays an important role in the autophagy pathway. Two key proteins of autophagy, LC3B and Beclin-1, were examined to assess autophagic activity. Protein expression of LC3B-I and LC3B-II (autophagosome marker proteins) was determined by Western blot analysis, and the LC3B-II/LC3B-I ratios were positively correlated with the number of autophagosomes (Kadowaki and Karim, 2009). Upon autophagy induction with rapamycin, the LC3B-II/LC3B-I ratio increased significantly by 2.9-4.7-fold (Fig. $3 \mathrm{~A}$ and Supplementary Fig. 3A). Expression of TIG1A in HtTA cells increased the LC3B-II/LC3B-I ratio by 5.62 -fold (Figs. 3A and 3B) In cells co-transfected with pTIG1A-myc and pTMEM192-Flag expression vectors and then treated with rapamycin, the ratio of LC3B-II to LC3B-I increased by 4.8-fold. Similarly, in cells expressing TIG1B the ratio of LC3B-II to LC3B-I was increased by $136 \%$, and the ratio was increased by 5.8 -fold for rapamycin- 
treated HtTA cells co-expressing TIG1B and TMEM192 (Supplementary Figs. 3A and 3B). TMEM192 had no effect on the LC3B-II/LC3B-I ratio in HtTA cells. The production of cleaved caspase-3 was also analyzed as an indicator of cell apoptosis in cells experiencing lysosomal degradations. However, no cleaved caspase- 3 was observed in cells expressing the TIG1A or TIG1B protein (data not shown).
We further examined the effect of TIG1 on Beclin-1 production. The levels of Beclin-1 were increased by 1.72-2.26-folds in cells expressing TIG1A or TIG1B. In cells expressing TIG1A or TIG1B along with TMEM192 and treated with rapamycin, the levels of beclin-1 were increased by 2.1-7.6-folds (Fig. 3C and Supplementary Fig. 3C). Similar results were observed when the production of Beclin-1 was detected by ELISA. We found
A

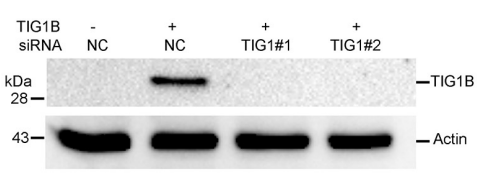

C

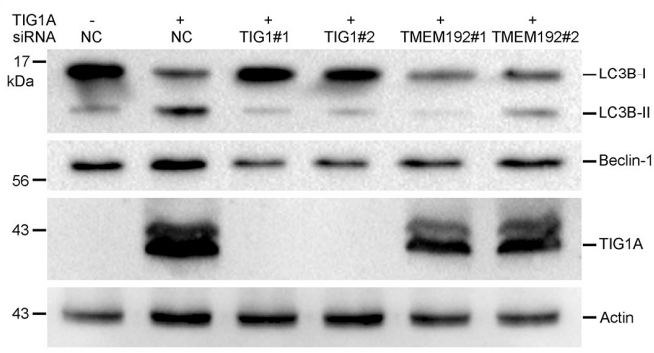

E

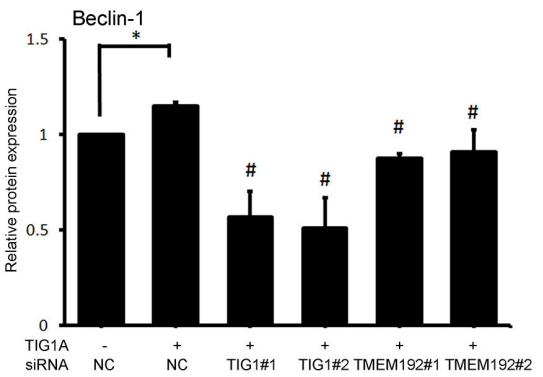

G

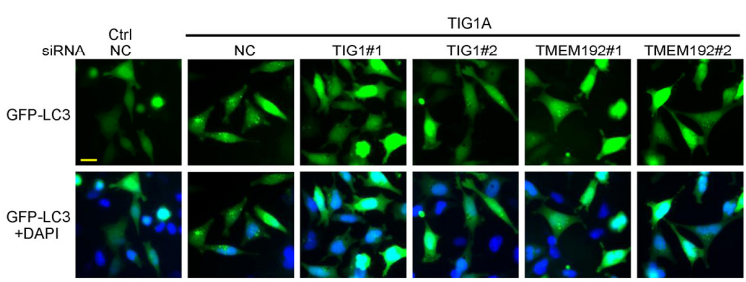

B

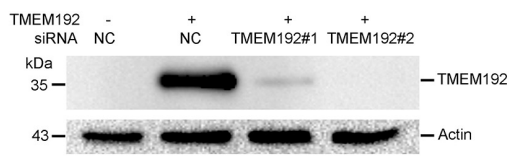

D

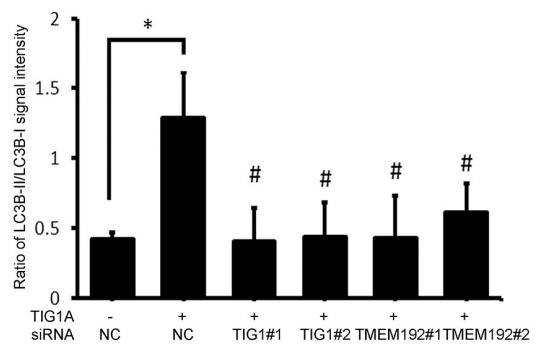

$\mathbf{F}$

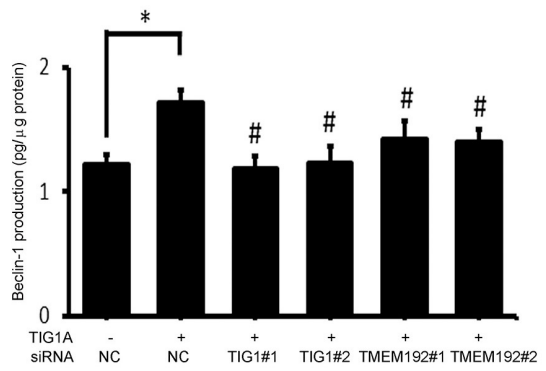

H

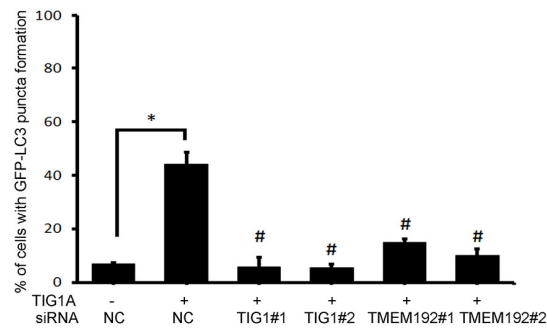

Fig. 4. TMEM192 siRNAs alleviated TIG1A-induced expression of autophagy-related proteins. HtTA cells plated in 6-well dishes were transfected with $0.5 \mu \mathrm{g}$ of TIG1B-myc (A) or TMEM192-Flag (B) expression vector along with $30 \mathrm{nM}$ TIG1, TMEM192, or NC siRNA for $48 \mathrm{~h}$. Alternatively, cells were transfected with $0.5 \mu \mathrm{g}$ of TIG1A-myc (C-E) expression vector along with the indicated siRNA (30 nM) for $48 \mathrm{~h}$ and were then cultured in serum-free medium for $6 \mathrm{~h}$. Cell lysates were prepared, and the expression of TIG1, TMEM192, Beclin-1, or LC3B was determined using anti-MYC, anti-FLAG, anti-Beclin-1, or anti-LC3B antibodies, respectively. Experimental results are summarized as the mean percentage $( \pm S D)$ of the ratio of LC3B-II to LC3B-I and the level of Beclin-1 with each sample normalized to the level of actin protein in two independent experiments (D, E). HtTA cells plated in 6-well dishes were transfected with $0.5 \mu \mathrm{g}$ of TIG1A-myc expression vectors along with the indicated siRNA for $48 \mathrm{~h}$ and were then cultured in serum-free medium for $6 \mathrm{~h}$. Cell lysates were prepared, and the level of Beclin-1 was detected using an enzyme immunoassay. Representative results of three independent experiments are shown. HtTA cells plated in triplicate in 24-well plates were transfected with $75 \mathrm{ng}$ of TIG1A-myc expression vector, $150 \mathrm{ng}$ pGFP-LC3 expression vector and with the indicated 30 $\mathrm{nM}$ siRNA for $48 \mathrm{~h}$ and were then cultured in serum-free medium for $6 \mathrm{~h}$. Representative images with GFP-LC3 puncta formation (G). Bar chart indicating the percentage of cells with GFP-LC3 puncta formation from three independent experiments $(\mathrm{H})$. Scale bar, $10 \mu \mathrm{m}$. *Indicates $p$ value $<0.05$. "Indicates $p$ value $<0.05$ when cells were co-transfected with TIG1A expression vector and indicated siRNA compared to cells that were co-transfected with TIG1A expression vector and NC siRNA. 
that expression of TIG1A and TIG1B increased Beclin-1 production in HtTA cells by $25 \%$ and $54 \%$, respectively (Fig. 3D and Supplementary Fig. 3D). Rapamycin treatment of HtTA cells increased Beclin-1 production by $18.9-27 \%$ when cells were co-transfected with pTIG1A-myc or pTIG1B-myc expression vector and pTMEM192-Flag vector compared with cells transfected with only pTIG1A-myc or pTIG1B-myc expression vector.

Cells transfected to express GFP-LC3 showed autophagosome formation in response to rapamycin (Fig. 3E and Supplementary Fig. 3E). GFP-LC3 puncta formation was increased in cells expressing TIG1A or TIG1B. Rapamycin-treated HITA cells co-expressing TIG1A or TIG1B with TMEM192 show more puncta, suggesting that autophagosome formation was markedly increased when TIG1 and TMEM192 were present
(Fig. 3F and Supplementary Fig. 3F).

SQSTM1/p62 is an autophagy adaptor. The levels of SQSTM1/p62 decreased in cells treated with rapamycin but not in cells expressing TIG1A or TIG1B. This result suggests that TIG1 does not enhance cell autophagy through the SQSTM1/ p62 protein (Fig. 3A, Supplementary Figs. 3A and 4).

\section{Silencing of TMEM192 alleviated the TIG1-induced expression of autophagy-related proteins}

To ascertain the role of TMEM192 in TIG1-mediated upregulation of autophagy, we examined the effects of TMEM192 silencing on the production of LC3B-II and Beclin-1 in TIG1expressing cells. HtTA cells were transiently transfected with pTIG1-myc or pTMEM192-Flag expression vector along with
A

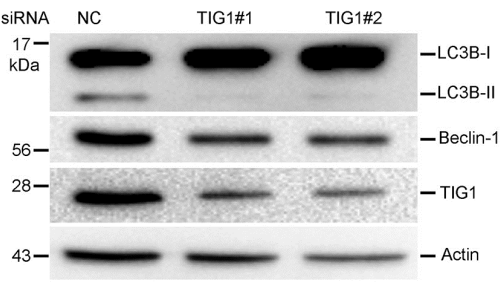

C

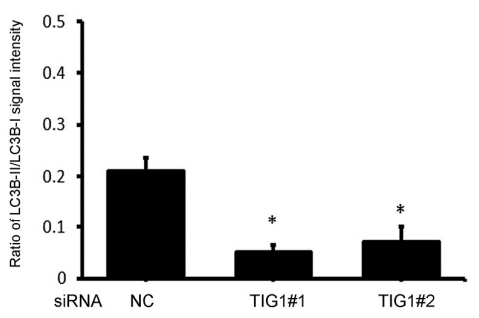

$\mathbf{E}$

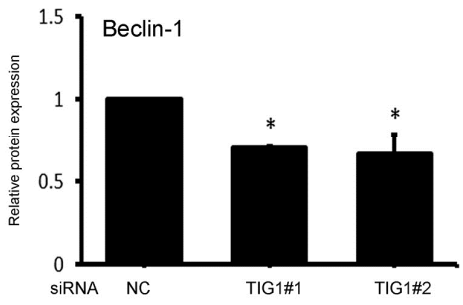

G

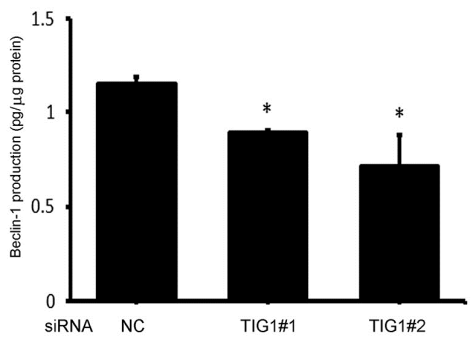

B

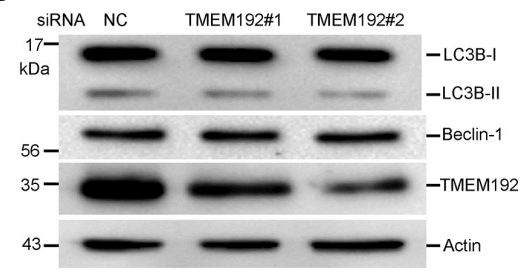

D

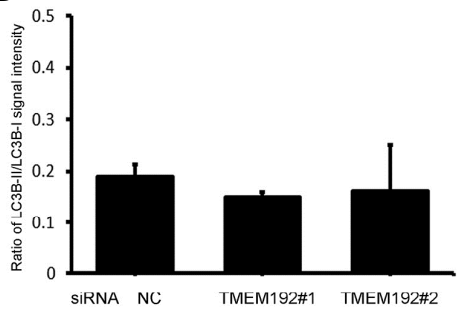

$\mathbf{F}$

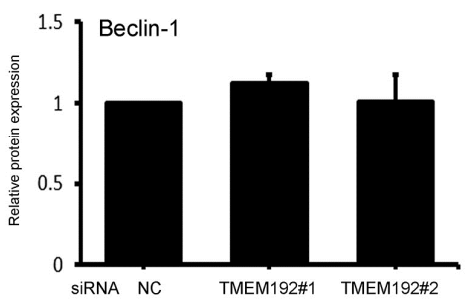

H

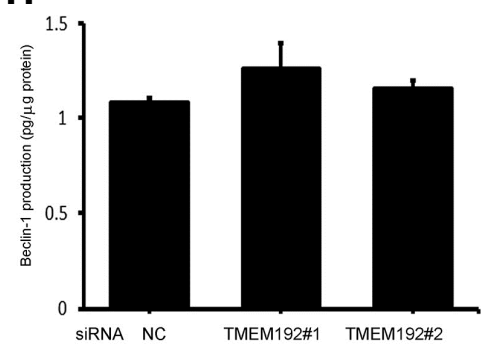

Fig. 5. TIG1 siRNAs suppressed the expression of autophagy-related proteins. HtTA cells plated in 6-well dishes were transfected with the indicated siRNA $(30 \mathrm{nM})$ for $48 \mathrm{~h}$ and were then cultured in serum-free medium for $6 \mathrm{~h}$. Cell lysates were prepared, and the expression of TIG1, TMEM192, or LC3B was determined using anti-TIG1 (A), anti-TMEM192 (B), or anti-LC3B and Beclin-1 (A, B) antibodies, respectively. Experimental results are summarized as the mean percentage $( \pm S D)$ of the ratio of LC3B-II to LC3B-I and the level of Beclin-1 with each sample normalized to the level of actin protein in two independent experiments (C-F). The level of Beclin-1 was detected using an enzyme immunoassay $(G, H)$. Representative results of three independent experiments are shown. *Indicates $p$ value $<0.05$. 
siRNAs specific for TIG1 or TMEM192 for $48 \mathrm{~h}$. No TIG1A or TIG1B protein was detected in TIG1A or TIG1B-expressing cells transfected with each TIG1 siRNA, while TMEM192 protein levels were reduced by $87 \%$ or were barely detectable in TMEM192-expressing cells transfected with each TMEM192 siRNA (Figs. 4A-4C).

Our results showed that the LC3B-II/LC3B-I ratios were markedly decreased by $66.2-68.2 \%$ or $52.6-66.5 \%$ in TIG1Aexpressing cells co-transfected with either TIG1 or TMEM192 siRNAs compared with cells co-transfected with NC siRNA (Figs. 4C and 4D). Similarly, compared to TIG1B-expressing cells transfected with NC siRNA, TIG1 or TMEM192 siRNAs significantly decreased the LC3B-II/LC3B-I ratios by 77.8 $80.5 \%$ or $51.7-74 \%$, respectively (Supplementary Figs. $5 \mathrm{~A}$ and 5B). We also examined the effect of TIG1 or TMEM192 siRNAs on TIG1-induced Beclin-1 production. We found that Beclin-1 production was decreased by $28.3-55.7 \%$ and $17.5-23.8 \%$ in TIG1A-expressing HtTA cells co-transfected with either TIG1 or TMEM192 siRNAs compared with cells co-transfected with NC siRNA (Figs. 4E and 4F). Similarly, TIG1 or TMEM192 siRNAs also inhibited Beclin-1 production by $27.9-50.8 \%$ and 20.8$56.4 \%$, respectively, in TIG1B-expressing cells (Supplementary
Figs. 5C and 5D). To clarify the effects of TIG1 on Beclin-1, we also examined the mRNA expression of Beclin-1. Our results showed that the expression of Beclin-1 mRNA was significantly increased by 12.8-17.8-fold in TIG1-expressing HtTA cells. TIG1 or TMEM192 siRNAs abolished the increased expression of Beclin-1 mRNA (Supplementary Fig. 6). In addition, the increased GFP-LC3 puncta formation was alleviated in cells expressing TIG1 and TMEM192 siRNAs (Figs. 4G, 4H, Supplementary Figs. 5E and 5F).

\section{Silencing TIG1 suppressed the expression of autophagy-} related proteins

To further confirm the enhanced ability of TIG1 to influence autophagy, we examined the effect of endogenous TIG1 on the production of LC3B and Beclin-1. TIG1B, but not TIG1A, could be detected in HtTA and Huh7 cells using TIG1 antibody (Fig.5A and Supplementary Fig. 7A). We observed that transient expression of TIG1 siRNAs in HtTA cells for $48 \mathrm{~h}$ led to downregulation of TIG1 ranging from $60-84.4 \%$, while TMEM192 siRNAs reduced TMEM192 levels by $20.4-57.8 \%$ (Figs. 5A and 5B). Our results showed that the ratio of LC3B-II to LC3B-I and the production of Beclin-1 were decreased by
A

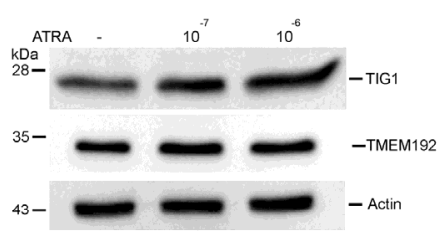

C

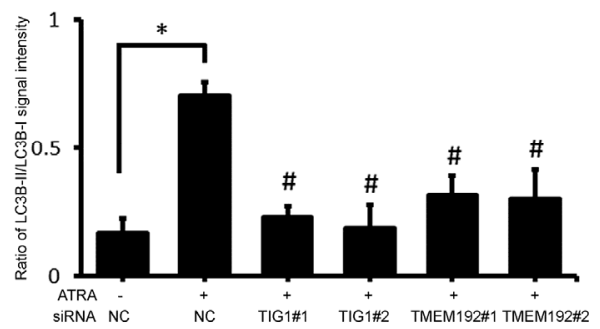

$\mathbf{E}$

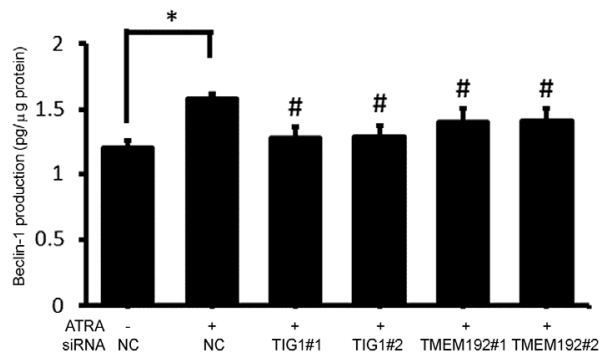

B

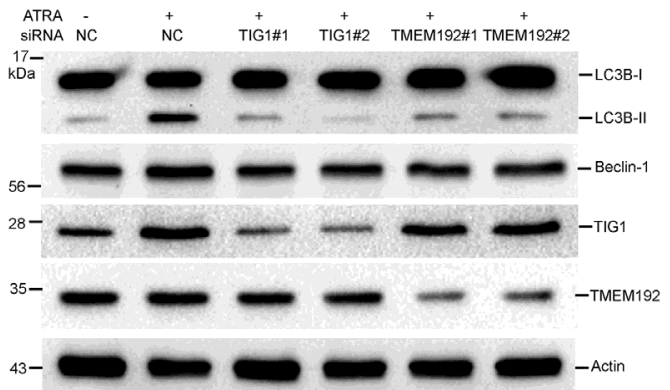

D

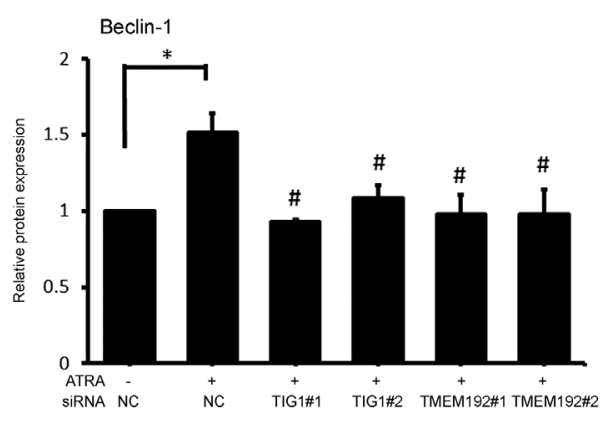

Fig. 6. TIG1 and TMEM192 siRNAs decrease ATRA-induced upregulation of autophagy. HITA cells plated in 6-well dishes were treated daily with the indicated concentration of ATRA for $48 \mathrm{~h}(\mathrm{~A})$. Alternatively, cells were transfected with the indicated siRNA $(30 \mathrm{nM})$ and then treated daily with $10^{-6} \mathrm{M}$ ATRA for $48 \mathrm{~h}$. Cells were then cultured in serum-free medium for $6 \mathrm{~h}(\mathrm{~B}-\mathrm{D})$. Cell lysates were prepared, and the expression of TIG1, TMEM192, Beclin-1, or LC3B was determined using anti-TIG1, anti-TMEM192, Beclin-1, or anti-LC3B antibodies (B), respectively. Experimental results are summarized as the mean percentage $( \pm S D)$ of the ratio of LC3B-II to LC3B-I and the level of Beclin-1 with each sample normalized to the level of actin protein in two independent experiments (C, D). The level of Beclin-1 was detected using an enzyme immunoassay (E). Representative results of three independent experiments are shown. *Indicates $p$ value $<0.05$. "Indicates $p$ value $<0.05$ when cells were transfected with the indicated siRNA and then treated with ATRA compared to cells transfected with NC siRNA and then treated with ATRA. 
66-75.3\% and $22.7-37.9 \%$, respectively, in TIG1 siRNAtransfected cells (Figs. 5A, 5C, 5E, and 5G). Neither the ratio of LC3B-II to LC3B-I nor the production of Beclin-1 was affected in HtTA cells transfected with TMEM192 siRNAs (Figs. 5B, 5D, $5 \mathrm{~F}$, and $5 \mathrm{H}$ ). Similar inhibitory effects on the production of LC3B and Beclin-1 were observed in Huh7 cells transfected with TIG1 siRNAs (Supplementary Fig. 7).

Silencing TIG1 and TMEM192 expression decreases the ATRA-induced upregulation of autophagy

TIG1 is a retinoid-inducible protein (Nagpal et al., 1996), and previous studies have confirmed that ATRA can induce the activation of autophagy in leukemia cells (Anguiano et al., 2013; Orfali et al., 2015; Rajawat et al., 2010). To determine whether TIG1 or TMEM192 contributes to the ATRA-mediated activation of autophagy, we examined the effects of TIG1 or TMEM192 silencing on the production of LC3B and Beclin-1 in ATRA-treated cells. As shown in Fig. 6A, TIG1 levels were increased in a dose-dependent manner, with a maximal $45 \%$ increase in cells treated with $10^{-6} \mathrm{M}$ ATRA (Fig. 6A). Our results showed that the ratio of LC3B-II to LC3B-I and the production of Beclin-1 were markedly increased by 3.2-fold and 31.5$64.3 \%$, respectively, in cells treated with $10^{-6} \mathrm{M}$ ATRA (Figs. $6 \mathrm{~B}$, $6 \mathrm{C}, 6 \mathrm{D}$, and $6 \mathrm{E})$. The ratio of LC3B-II to LC3B-I and the production of Beclin-1 were decreased by $55.1-73.5 \%$ and $10.7-$ $38.4 \%$, respectively, in ATRA-treated HtTA cells that expressed either TIG1 or TMEM192 siRNAs compared with cells expressing NC siRNA (Figs. 6B, 6C, 6D, and 6E). No obvious change of TIG1 or TMEM192 protein levels was observed in cells transfected with TMEM192 or TIG1 siRNAs, respectively (Fig. 6B). The result confirms that expression of TIG1 or TMEM192 in HtTA cells did not appear to influence each other protein level observed in Fig. 2. Similar effects on LC3B and Beclin-1 were observed in ATRA-treated Huh7 cells transfected with TIG1 and TMEM192 siRNAs (Supplementary Fig. 8). In addition to autophagic activity measured by the ratio of LC3B-II to LC3B-I and the production of Beclin-1, a similar result was observed for GFP-LC3 puncta formation in ATRA-treated HtTA cells coexpressing TIG1 or TMEM192 siRNAs (Supplementary Fig. 9).

\section{DISCUSSION}

TIG1 is thought to be a retinoid-inducible tumor suppressor, and its mechanism of tumor suppression is largely unknown. Our previous studies showed that both isoforms of TIG1 suppressed cell growth and induced $G$ protein-coupled receptor kinase 5 (GRK5) expression in colon cancer cells (Wu et al., 2011). We further explored the inhibitory effect of TIG1 on PGE2-induced cellular proliferation and signaling in colon cancer cells, which are processes that involve the downregulation of $G$ protein-coupled signaling via the upregulation of GRK5 expression (Tsai et al., 2011). In addition to the upregulation of GRK5 by TIG1, we demonstrated here that TIG1 interacted with TMEM192 to induce autophagic activity in HtTA cervical cells and Huh7 hepatic cells.

Autophagy has been reported to be involved in cancer cell proliferation or tumorigenesis in a variety of ways (White, 2015). The Beclin-1 autophagy gene is monoallelically deleted in 40$75 \%$ of human sporadic breast, ovarian, and prostate cancer (Aita et al., 1999; Choi et al., 2013; Liang et al., 1999). Heterozygous disruption of Beclin-1 increases the frequency of liver and lung tumors and lymphomas in mice (Qu et al., 2003; Yue et al., 2003). Cancer cells also rely on autophagy. Autophagy is upregulated in RAS-transformed cancer cells and facilitates cancer growth and tumorigenesis (Guo et al., 2011; Lock et al., 2011; 2014). Mice with systemic mosaic deletion of Atg5 or Atg7 develop benign liver adenomas (Takamura et al., 2011). Autophagy has a critical role in pancreatic ductal adenocarcinoma pathogenesis (Yang et al., 2011). Therefore, autophagy promotes or inhibits tumor progression depending on the cell types and the microenvironment stimuli. The underlying mechanisms by which TIG1 induces autophagy are not completely clear at present. In addition to the target TMEM192 protein, the involvement of the TIG1/TMEM192 complex in autophagosome formation and activation of autophagy needs further investigation. Furthermore, the role of induced autophagic activity by TIG1 in cancer cell proliferation or tumorigenesis should also be determined.

Aside from its involvement in cancer development, autophagy plays a critical role in a variety of cell differentiation processes (Bernard et al., 2014; Simon, 2012; Vessoni et al., 2012; Zhuang et al., 2011). Autophagy regulates myeloid cell differentiation, and autophagy is increased during ATRA-induced granulocytic differentiation of acute promyelocytic leukemia cells (Orfali et al., 2015; Wang et al., 2011). How autophagy is induced by ATRA is not yet known, but our results revealed that silencing of either TIG1 or TMEM192 alleviated ATRAupregulated autophagy. Whether the TIG1/TMEM192 pathway is involved in ATRA-mediated myeloid cell differentiation needs further investigation. Regardless of how the downstream signaling pathway of TIG1 is regulated, TMEM192 knockdown affects TIG1-mediated upregulation of autophagy.

Although TIG1-mediated autophagy depends on the expression of TMEM192 as indicated by the present study, it has also been shown that TMEM192 deficiency triggers autophagy in HepG2 hepatoma cells (Liu et al., 2012). Furthermore, based on caspase-12 activation, apoptosis was not observed in HtTA cervical cells in which TMEM192 was either overexpressed or silenced (data not shown). Difference in cell type might be the main cause of the different effects elicited by TMEM192 deficiency. Enhanced TMEM192 can only mediate TIG1 induction of autophagy-related proteins in rapamycin-treated TIG1expressing cells but not in untreated TIG1-expressing cells (Fig. 3 and Supplementary Fig. 3). Knockdown of TMEM192 did not inhibit cell autophagy (Fig. 5B and Supplementary Fig. 7) suggests that remaining TMEM192 is sufficient to maintain the autophagic activity. These results suggest that endogenous TMEM192 is capable of TIG1-mediated autophagic activity but is insufficient to induce an autophagy response simply due to environmental stress. Conversely, knockdown of TMEM192 can reduce TIG1-mediated upregulation of autophagy.

Our present results demonstrate that only the TIG1B isoform was detected in HtTA and Huh7 cells, even though similar mRNA expression of TIG1A and TIG1B was observed (Wu et al., 2011). Similarly, previous studies analyzing the suppression of cell growth and invasion by TIG1 have focused on the TIG1B isoform (Jing et al., 2002; Kwok et al., 2009). Regardless of the different expression levels of TIG1A and TIG1B, both TIG1 isoforms exhibited similar autophagic activities in transiently transfected HtTA cervical cells. This result suggests that the induction of autophagic activities by the TIG1 protein may reside in the amino terminus region of the TIG1 protein, which is supported by co-localization and interaction of both of the TIG1 isoforms with TMEM192 and the identical effect of the TIG1 isoforms on TMEM192-mediated regulation of autophagyrelated proteins. Further analysis of the correlation between TMEM192 and Atg activities will be important for elucidation of the autophagic function of the TIG1 proteins. 
In conclusion, our present results demonstrate that TIG1 isoforms can interact with TMEM192. Expression of TIG1 led to autophagy, and silencing of TMEM192 resulted in decreased autophagic activity induced by TIG1. Furthermore, silencing of either TIG1 or TMEM192 expression decreased the ATRAmediated upregulation of autophagy. Our results show that TIG1 is involved in ATRA-induced autophagic activity via the TMEM192 protein.

Note: Supplementary information is available on the Molecules and Cells website (www.molcells.org).

\section{ACKNOWLEDGMENTS}

This work was supported by a grant (TCRD-TPE-105-17) from the Taipei Tzuchi Hospital through the Buddhist Tzuchi Medical Foundation, Taipei, Taiwan. The authors thank the Core Laboratory of the Buddhist Tzuchi General Hospital for support.

\section{REFERENCES}

Aagaard, A., Listwan, P., Cowieson, N., Huber, T., Ravasi, T., Wells, C.A., Flanagan, J.U., Kellie, S., Hume, D.A., Kobe, B., et al. (2005). An inflammatory role for the mammalian carboxypeptidase inhibitor latexin: relationship to cystatins and the tumor suppressor TIG1. Structure 13, 309-317.

Aita, V.M., Liang, X.H., Murty, V.V., Pincus, D.L., Yu, W., Cayanis, E., Kalachikov, S., Gilliam, T.C., and Levine, B. (1999). Cloning and genomic organization of beclin 1, a candidate tumor suppressor gene on chromosome 17q21. Genomics 59, 59-65.

Anguiano, J., Garner, T.P., Mahalingam, M., Das, B.C., Gavathiotis, E., and Cuervo, A.M. (2013). Chemical modulation of chaperonemediated autophagy by retinoic acid derivatives. Nat. Chem. Biol. 9, 374-382.

Bernard, M., Dieude, M., Yang, B., Hamelin, K., Underwood, K., and Hebert, M.J. (2014). Autophagy fosters myofibroblast differentiation through MTORC2 activation and downstream upregulation of CTGF. Autophagy 10, 2193-2207.

Chen, X.H., Wu, W.G., and Ding, J. (2014). Aberrant TIG1 methylation associated with its decreased expression and clinicopathological significance in hepatocellular carcinoma. Tumour Biol. 35, 967-971.

Choi. A.M., Ryter, S.W., and Levine, B. (2013). Autophagy in human health and disease. N. Engl. J. Med. 368, 651-662.

Dooley, H.C., Razi, M., Polson, H.E., Girardin, S.E., Wilson, M.I., and Tooze, S.A. (2014). WIPI2 links LC3 conjugation with PI3P, autophagosome formation, and pathogen clearance by recruiting Atg12-5-16L1. Mol. Cell 55, 238-252.

Guo, J.Y., Chen, H.Y., Mathew, R., Fan, J., Strohecker, A.M., Karsli-Uzunbas, G., Kamphorst. J.J., Chen, G., Lemons, J.M., Karantza, V., et al. (2011). Activated Ras requires autophagy to maintain oxidative metabolism and tumorigenesis. Genes Dev. $25,460-470$

Hanada. T., Noda, N.N., Satomi, Y., Ichimura, Y., Fujioka, Y., Takao, T., Inagaki, F., and Ohsumi, Y. (2007). The Atg12-Atg5 conjugate has a novel E3-like activity for protein lipidation in autophagy. J. Biol. Chem. 282, 37298-37302.

Hara, T., Takamura, A., Kishi, C., lemura, S., Natsume, T., Guan, J.L., and Mizushima, N. (2008). FIP200, a ULK-interacting protein, is required for autophagosome formation in mammalian cells. J. Cell Biol. 181, 497-510.

Jing, C., El-Ghany, M.A., Beesley, C., Foster, C.S., Rudland, P.S., Smith, P., and Ke, Y. (2002). Tazarotene-induced gene 1 (TIG1) expression in prostate carcinomas and its relationship to tumorigenicity. J. Natl. Cancer Inst. 94, 482-490.

Kadowaki, M., and Karim, M.R. (2009). Cytosolic LC3 ratio as a quantitative index of macroautophagy. Methods Enzymol. 452, 199-213.

Kang, R., Zeh, H.J., Lotze, M.T., and Tang, D. (2011). The Beclin 1 network regulates autophagy and apoptosis. Cell Death Differ. 18, 571-580.

Kobayashi, S. (2015). Choose delicately and reuse adequately: the newly revealed process of autophagy. Biol. Pharm. Bull. 38,
1098-1103.

Kwok, W.K., Pang, J.C., Lo, K.W., and Ng, H.K. (2009). Role of the RARRES1 gene in nasopharyngeal carcinoma. Cancer Genet. Cytogenet. 194, 58-64.

Kwong. J., Lo, K.W., Chow, L.S., Chan, F.L., To, K.F., and Huang, D.P. (2005). Silencing of the retinoid response gene TIG1 by promoter hypermethylation in nasopharyngeal carcinoma. Int. $\mathrm{J}$. Cancer 113, 386-392.

Lamb, C.A., Yoshimori, T., and Tooze, S.A. (2013). The autophagosome: origins unknown, biogenesis complex. Nat. Rev. Mol. Cell Biol. 14, 759-774.

Lee, I.H., Cao, L., Mostoslavsky, R., Lombard, D.B., Liu, J., Bruns, N.E., Tsokos, M., Alt, F.W., and Finkel, T. (2008). A role for the NAD-dependent deacetylase Sirt1 in the regulation of autophagy. Proc. Natl. Acad. Sci. USA 105, 3374-3379.

Lee, J., Giordano, S., and Zhang, J. (2012). Autophagy, mitochondria and oxidative stress: cross-talk and redox signalling Biochem. J. 441, 523-540.

Liang, X.H., Jackson, S., Seaman, M., Brown, K., Kempkes, B., Hibshoosh, H., and Levine, B. (1999). Induction of autophagy and inhibition of tumorigenesis by beclin 1. Nature 402, 672-676.

Liang, Y., Jansen, M., Aronow, B., Geiger, H., and Van Zant, G. (2007). The quantitative trait gene latexin influences the size of the hematopoietic stem cell population in mice. Nat. Genet. 39, 178-188

Liu, Z., Lv, Y.J., Song, Y.P., Li, X.H., Du, Y.N., Wang, C.H., and Hu, L.K. (2012). Lysosomal membrane protein TMEM192 deficiency triggers crosstalk between autophagy and apoptosis in HepG2 hepatoma cells. Oncol. Rep. 28, 985-991.

Lock, R., Roy, S., Kenific, C.M., Su, J.S., Salas, E., Ronen, S.M., and Debnath, J. (2011). Autophagy facilitates glycolysis during Ras-mediated oncogenic transformation. Mol. Biol. Cell. 22, 165178.

Lock, R., Kenific, C.M., Leidal, A.M., Salas, E., and Debnath, J. (2014). Autophagy-dependent production of secreted factors facilitates oncogenic RAS-driven invasion. Cancer Discov. 4 466-479.

Mizuiri, H., Yoshida, K., Toge, T., Oue, N., Aung, P.P., Noguchi, T., and Yasui, W. (2005). DNA methylation of genes linked to retinoid signaling in squamous cell carcinoma of the esophagus: DNA methylation of CRBP1 and TIG1 is associated with tumor stage. Cancer Sci. 96, 571-577.

Mizushima, N., and Komatsu, M. (2011). Autophagy: renovation of cells and tissues. Cell 147, 728-741.

Mizushima, N., Yoshimori, T., and Ohsumi, Y. (2011). The role of Atg proteins in autophagosome formation. Annu. Rev. Cell Dev. Biol. 27, 107-132.

Nagpal, S., Patel, S., Asano, A.T., Johnson, A.T., Duvic, M., and Chandraratna, R.A. (1996). Tazarotene-induced gene 1 (TIG1), a novel retinoic acid receptor-responsive gene in skin. J. Invest. Dermatol. 106, 269-274.

Orfali, N., O'Donovan, T.R., Nyhan, M.J., Britschgi, A., Tschan, M.P., Cahill, M.R., Mongan, N.P., Gudas, L.J., and McKenna, S.L. (2015). Induction of autophagy is a key component of alltrans-retinoic acid-induced differentiation in leukemia cells and a potential target for pharmacologic modulation. Exp. Hematol. 43 , 781-793 e782.

Peng, Z., Shen, R., Li, Y.W., Teng, K.Y., Shapiro, C.L., and Lin, H.J. (2012). Epigenetic repression of RARRES1 is mediated by methylation of a proximal promoter and a loss of CTCF binding Plos One 7, e36891.

Qu, X., Yu, J., Bhagat, G., Furuya, N., Hibshoosh, H., Troxel, A., Rosen, J., Eskelinen, E.L., Mizushima, N., Ohsumi, Y., et al. (2003). Promotion of tumorigenesis by heterozygous disruption of the beclin 1 autophagy gene. J. Clin. Invest. 112, 1809-1820.

Rajawat, Y., Hilioti, Z., and Bossis, I. (2010). Autophagy: a target for retinoic acids. Autophagy 6,1224-1226.

Russell, R.C., Tian, Y., Yuan, H., Park, H.W., Chang, Y.Y., Kim, J., Kim, H., Neufeld, T.P., Dillin, A., and Guan, K.L. (2013). ULK1 induces autophagy by phosphorylating Beclin-1 and activating VPS34 lipid kinase. Nat. Cell Biol. 15, 741-750.

Schroder, B., Wrocklage, C., Hasilik, A., and Saftig, P. (2010). Molecular characterisation of 'transmembrane protein 192 ' (TMEM192), a novel protein of the lysosomal membrane. Biol. Chem. 391, 695-704.

Shutoh, M., Oue, N., Aung, P.P., Noguchi, T., Kuraoka, K., 
Nakayama, H., Kawahara, K., and Yasui, W. (2005). DNA methylation of genes linked with retinoid signaling in gastric carcinoma: expression of the retinoid acid receptor beta, cellular retinol-binding protein 1 , and tazarotene-induced gene 1 genes is associated with DNA methylation. Cancer 104, 1609-1619.

Simon, H.U. (2012). Autophagy in myocardial differentiation and cardiac development. Circ. Res. 110, 524-525.

Takamura, A., Komatsu, M., Hara, T., Sakamoto, A., Kishi, C. Waguri, S., Eishi, Y., Hino, O., Tanaka, K., and Mizushima, N. (2011). Autophagy-deficient mice develop multiple liver tumors. Genes Dev. 25, 795-800.

Tsai, F.M., Wu, C.C., Shyu, R.Y., Wang, C.H., and Jiang, S.Y. (2011). Tazarotene-induced gene 1 inhibits prostaglandin E2stimulated HCT116 colon cancer cell growth. J. Biomed. Sci. 18, 88

Vessoni, A.T., Muotri, A.R., and Okamoto, O.K. (2012). Autophagy in stem cell maintenance and differentiation. Stem Cells Dev. 21, 513-520.

Wang, Z., Cao, L., Kang, R., Yang, M., Liu, L., Zhao, Y., Yu, Y., Xie, M., Yin, X., Livesey, K.M., et al. (2011). Autophagy regulates myeloid cell differentiation by p62/SQSTM1-mediated degradation of PML-RARalpha oncoprotein. Autophagy 7, 401411.

White, E. (2015). The role for autophagy in cancer. J. Clin. Invest. $125,42-46$.

Wu, C.C., Shyu, R.Y., Chou, J.M., Jao, S.W., Chao, P.C., Kang, J.C., Wu, S.T., Huang, S.L., and Jiang, S.Y. (2006). RARRES1 expression is significantly related to tumour differentiation and staging in colorectal adenocarcinoma. Eur. J. Cancer 42, 557565.

Wu, C.C., Tsai, F.M., Shyu, R.Y., Tsai, Y.M., Wang, C.H., and Jiang, S.Y. (2011). G protein-coupled receptor kinase 5 mediates Tazarotene-induced gene 1-induced growth suppression of human colon cancer cells. BMC Cancer 11, 175.

Yanatatsaneejit, P., Chalermchai, T., Kerekhanjanarong, V., Shotelersuk, K., Supiyaphun, P., Mutirangura, A., and Sriuranpong, V. (2008). Promoter hypermethylation of CCNA1, RARRES1, and HRASLS3 in nasopharyngeal carcinoma. Oral Oncol. 44, 400-406.

Yang, S., Wang, X., Contino, G., Liesa, M., Sahin, E., Ying, H., Bause, A., Li, Y., Stommel, J.M., Dell'antonio, G., et al. (2011) Pancreatic cancers require autophagy for tumor growth. Genes Dev. 25, 717-729.

Yue, Z., Jin, S., Yang, C., Levine, A.J., and Heintz, N. (2003). Beclin 1 , an autophagy gene essential for early embryonic development is a haploinsufficient tumor suppressor. Proc. Natl. Acad. Sci. USA 100, 15077-15082.

Zhang, J., Liu, L., and Pfeifer, G.P. (2004). Methylation of the retinoid response gene TIG1 in prostate cancer correlates with methylation of the retinoic acid receptor beta gene. Oncogene 23 2241-2249.

Zhuang, W., Li, B., Long, L., Chen, L., Huang, Q., and Liang, Z. (2011). Induction of autophagy promotes differentiation of gliomainitiating cells and their radiosensitivity. Int. J. Cancer 129, 27202731. 